\title{
FAVOURABLE INTRACRANIAL TUMOURS \\ The Fifteenth Alexander Simpson Smith Memorial Lecture
}

\author{
(Delivered at the West London Hospital, September 4, 1962)
}

\author{
Leslie Oliver, M.B., B.S., F.R.C.S., F.A.C.S. \\ Neurosurgeon, Charing Cross Hospital, West London Hospital, Royal Northern Hospital and West End Hospital \\ for Neurology and Neurosurgery, London
}

IF the diagnosis of an intracranial tumour is made soon enough and the tumour is benign, a favourable outcome can nowadays be expected after operation. Therefore the clinician should be familiar with the whole range of benign tumours, and when patients present with evidence of a space-occupying lesion, its pathological nature must be proven beyond doubt. In most cases, this necessitates a biopsy carried out through a burr-hole or trephine opening. Benign tumours may occur anywhere within the skull. Although tumours often transgress the anatomical boundaries, it is of practical value to consider them in relation to the regions of the brain; frontal, temporal, parietal-occipital and cerebellar.

\section{Tumours of the Frontal Region}

Tumours in this region give rise to personality changes which may be obvious to the clinician, or may be revealed only after searching enquiry. More important facts may be elicited from near relations than from patients. Apathy is the keynote of the syndrome. Thus there may be loss of interest in work or hobbies. There is also a special type of incontinence often associated with a frontal lobe lesion: patients although fully alert may shamelessly pass urine and fæces anywhere (Jefferson, 1950). Mental euphoria is a common feature; thus patients may not be impressed by the seriousness of their condition, and tend to regard the prospect of a major operation with unusual equanimity. A generalized blunting of mental processes may occur, including impairment of memory for recent events; this is, however, sometimes observed with tumours in other situations. Tumours in the dominant frontal lobe may cause motor aphasia.

Tumours extending backwards in the frontal region involve the motor cortex or its efferents and thus produce contralateral hemiparesis. Epilepsy, generalized or focal, is a common manifestation of frontal tumours. Some of them produce a grasp reflex on the opposite side, the fingers automatically closing around objects placed in the hand. Contralateral hypotonia and incoordination sometimes occur, and occasionally there is nystagmus. This is of considerable practical importance, for confusion with tumours of the cerebellum may result. Signs of increased intracranial pressure, headache, vomiting and papilloedema, are common with tumours in the frontal region, but it is important to realize that such signs may be absent with tumours anywhere in the cranium.

Anteriorly placed tumours of the temporal region do not affect the forward loop of the optic radiation, and may therefore be confused with tumours of the frontal region. Hypertensive encephalopathy may simulate a frontal tumour. It is suggested by the presence of vascular hypertension, characteristic retinopathy, cardiac enlargement and albuminuria. However, a patient with these signs is not excluded from having a cerebral tumour. The dementia of Pick's presenile cerebral atrophy is likely to be more advanced than with frontal tumours, except those which involve the corpus callosum. Patients with a positive Wassermann and evidence of a spaceoccupying lesion, should be completely investigated as tumour suspects, and it is noteworthy that cerebral gummas rarely respond to antisyphilitic treatment (Bailey, 1948).

\section{Investigations}

Lumbar puncture should not be carried out if there is evidence of raised intracranial pressure caused by a space-occupying lesion, for it may precipitate or aggravate brain-stem compression, sometimes with a fatal result.

Electroencephalography, being in no way disturbing to patients, is carried out as a routine in the investigation of suspected intracranial tumours. It sometimes gives localizing evidence but supplementary investigation is always required.

Radiography. As with tumours elsewhere in the cranium, plain radiographs may show evidence of raised intracranial pressure (e.g. rarefaction or erosion of the posterior clinoid processes and dorsum sellæ). Abnormal vascular channels may be seen (Fig. I) and occasionally 


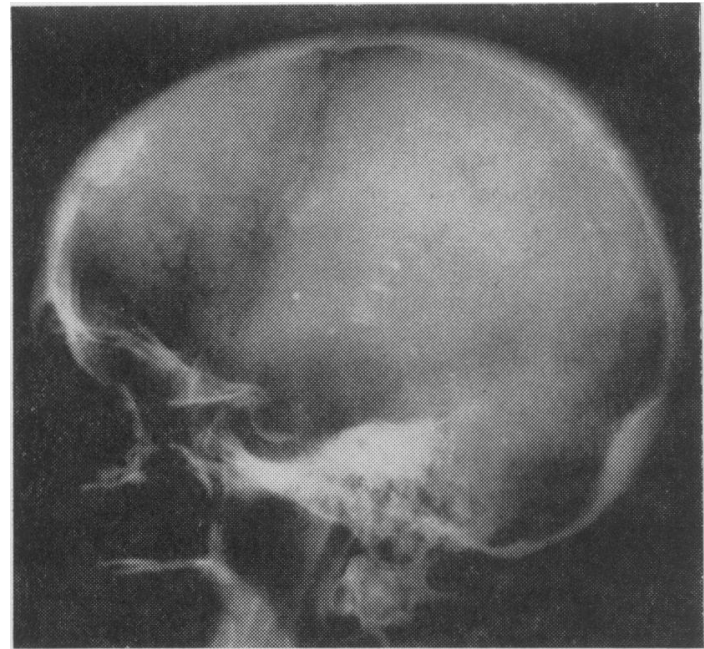

FIG. 1.-Abnormal vascular channels in the frontal region running in the direction of an area of hyperostosis. There was an underlying meningioma.

calcification of a tumour is demonstrated. In angiograms, the anterior cerebral artery is often displaced towards the opposite side, and the middle cerebral artery and its branches may be deflected downward and outward. Parasagittal meningiomas tend to produce a semi-circular downward curve in the branches of the anterior cerebral artery as seen in lateral views (Fig. 2). Olfactory groove meningiomas cause backward displacement of the anterior cerebral arteries. A clearly circumscribed tumour pattern is produced by some meningiomas. In ventriculograms the antero-posterior view shows displacement of the ventricles towards the opposite side and deformity or absence of filling of the anterior horn on the affected side. The third ventricle usually remains in the same antero-posterior plane as the septum lucidum in contrast with the angulation of these two structures produced by temporal tumours.

I shall now discuss the types of benign tumours which occur in the frontal region. About $13 \%$ of all primary intracranial tumours are meningiomas and they are found more often in the frontal compartment than elsewhere in the cranium (Cushing and Eisenhardt, 1938). They are usually attached to the dura mater, but they are enveloped by the brain from which some of their blood supply may be derived. The majority of meningiomas are benign, but recur if their dural base is not completely excised. Meningiomas are thought to arise from arachnoid cells in the dura mater (Schmidt, 1902). Their growth appears sometimes to be initiated by trauma, for an overlying scar or fracture line may be found (Cushing and Eisenhardt, 1938). Some meningiomas cause hyperostosis whereas others cause destruction of adjacent bone. Often there is no bone reaction. The hyperostosis may be invaded

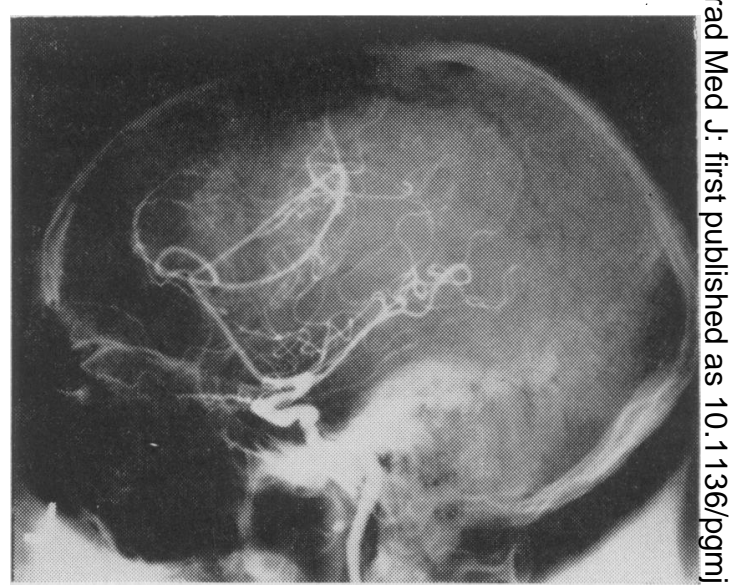

FIG. 2.-Downward curve of the calloso-marginal artery produced by a frontal parasagittal meningiomais The fronto-polar artery embraces the tumouf anteriorly and the carotid 'syphon' is compressef downwards. The patient complained of declining vision and headaches for three years.

윽

by tumour cells and should therefore be removeds at operation.

There are several histological varieties of meningioma (Russell, 1950). The commonest the endotheliomatous meningioma which is composed of alveolar masses or sheets of large polygo iato cells. There are often whorls of tumour ced 1 \& separated by bundles of reticulum fibres, ant small calcified bodies (calcospherites) may also be्? present. The term psammoma applies to meningiomas containing these calcified bodies which are sometimes dense enough to be revealecô radiologically. In the fibroblastic meningioma there are interlacing bundles of long spindle cellso containing fibroglial fibres. Whorls and cal $\frac{3}{5}$ cospherites are few. The angioblastic meningiom consists of masses of capillary spaces. The myxomatous meningioma is composed of a mixtures of spindle and stellate cells separated by mucio and reticulum fibrils.

Parasagittal meningiomas are those which aris from the superior sagittal (longitudinal) sinus 0 B falx. Some of them are bilateral. Ligation and excision of the affected part of the venous sinus is permissible when the tumour arises from the most anterior part of the sinus, but the posterio ligature must be well anterior to the veins draining the motor cortex. Further back, the tumour is removed as close to the sinus as possible and the्E tumour base coagulated with low diathermy॰ Meningiomas arising from the falx without in volving the superior longitudinal sinus can be completely removed with their dural attachment.

Olfactory groove meningiomas invariably cause anosmia on one or both sides. Backward extension 
may affect one or both optic nerves and sometimes the chiasm. When only one of the optic nerves is compressed there may be optic atrophy with loss of visual acuity on the affected side, and papillœdema on the other side (Foster-Kennedy syndrome). In the early stages of compression of an optic nerve, examination of the visual fields shows a central scotoma. There is a tendency for these tumours to grow downward into the nasal cavity. Plain radiography may show thickening of the floor of the anterior fossa, and angiography demonstrates upward and backward displacement of the anterior cerebral arteries. Olfactory groove meningiomas tend to envelop these arteries, which are therefore liable to injury at operation. The consequences may be serious. Clipping of one anterior cerebral artery can cause fatal arterial spasm or thrombosis. Survival after clipping both anterior cerebral arteries is most unlikely.

Suprasellar meningiomas arise from the tuberculum sellæ and grow upwards and backwards elevating the optic chiasm. Large tumours of this kind envelop the internal carotid arteries and their branches. The syndrome is characterized by bilateral diminishing visual acuity, optic atrophy and bitemporal hemianopia, but there is no ballooning of the pituitary fossa as with pituitary tumours. Plain radiography may, however, show hyperostosis of the tuberculum sellæ and erosion of the posterior clinoid processes. Ventriculography demonstrates deformity of the anterior end of the third ventricle. Bilateral carotid arteriography should be done to show the relationship of the arteries to these tumours. A bifrontal approach is indicated for suprasellar meningiomas. Any part of the capsule adherent to the internal carotid arteries and its branches, or the optic nerves and chiasm, should be left undisturbed.

\section{Benign Gliomas}

Truly benign astrocytomas (Grade I) are rare in the cerebrum as compared with their incidence in the cerebellum. They may appear to be circumscribed although tumour cells penetrate beyond the apparent plane of cleavage. These tumours may give rise to cysts. The fluid is yellow and contains a large amount of protein. The presence of a cyst is suggested by a sudden onset of symptoms or a fluctuating clinical course. A cyst may arise within an astrocytoma or on its outer surface. In the latter event, most of the cyst wall is smooth and free from tumour cells, and wide removal of the mural tumour prevents re-expansion of the cyst. A small proportion of these tumours, and especially those arising in the frontal pole and of the fibrillary type, are com- patible with long survival (Davis, Martin, Padberg and Anderson, I950).

Oligodendrogliomas, as the name suggests, are thought to be derived from oligodendrocytes, so named because of their paucity of dendritic processes. They are rare, with a maximum incidence in the fourth and fifth decades. They occur in the cerebral hemispheres, especially in the frontal lobes, more often than in any other part of the brain. They are composed of closely packed cells with spherical darkly staining nuclei, each surrounded by a ring or 'halo' of feebly staining cytoplasm. Calcification is common and can usually be demonstrated radiologically. They may be cystic. There is a good prospect of eight to I4 years' survival after wide excision (Earnest, Kernohan and Craig, 1950).

\section{Other Rare Tumours}

Epidermoid tumours (alternatively termed pearly tumours of Cruveilhier, or cholesteatomas) sometimes occur in the frontal region. They are distinguished from dermoid cysts by the absence of dermal appendages, i.e. hair follicles and sweat glands. There is a capsule of epidermal tissue only enclosing a mass of epithelial debris and cholesterol crystals. In ventriculograms, the air in the deformed ventricle has a characteristic mottled appearance (Oliver, 1952). All debris and crystals must be removed at operation otherwise an aseptic meningitis may occur, sometimes with a fatal outcome.

Colloid cysts may be conveniently considered now, for they are removed through a frontal exposure. They are thought to arise from paraphysial remnants, and are found attached to the choroid plexus in the third ventricle, obstructing the foramen of Monro. They are formed of a capsule of epithelial tissue and contain viscid colloid material. They cause attacks of hydrocephalus. Thus there are episodes of headache and vomiting, sometimes associated with papillœdema. Sometimes sudden loss of consciousness occurs. A history of recurrent weakness of the legs is occasionally obtained (Jefferson and Jackson, I939). Colloid cysts have a meagre attachment to the roof of the third ventricle and are easily removed through the anterior horn of the dilated lateral ventricle by means of an incision in the frontal cortex.

\section{Pituitary Tumours}

Pituitary tumours are also approached through the frontal region. Much of our knowledge of these tumours is derived from the monumental work of Harvey Cushing (Henderson, 1939).

Chromophobe adenomas are the pituitary tumours most frequently needing operative treatment. In 
this respect, they outnumber acidophil tumours by 28 to I (Pennybacker, 196I). They arise from the apparently functionless chromophobe cells of the anterior pituitary lobe. Cystic degeneration occurs more often than in acidophil adenomas. As these tumours grow they replace functioning cells and expand the pituitary fossa. Extension above the pituitary fossa brings them into contact with the optic chiasm and nerves, and the hypothalamus.

Patients are usually middle-aged, and the presenting symptom is declining vision. Examination reveals loss of visual acuity and often primary optic atrophy in one or both eyes. The classical and commonest visual field defect is bitemporal hemianopia which first affects the upper quadrants. This arises from the fact that although the tumours press on the chiasm from below, light rays falling on the lower part of the retina come from above. An homonymous hemianopia is sometimes found and indicates: pressure on the optic tract; a prefixed optic chiasm; or a large extension of the tumour into the temporal lobe. If one eye is blind and the other has a temporal field defect, originally there may have been either a bitemporal or an homonymous hemianopia. The history may help to decide this point. A central scotoma in one or both eyes without defects of the peripheral part of the fields may also indicate prefixation of the chiasm. The reason for this is not clear.

Upward and backward growth of a pituitary tumour indents the hypothalamus, but because the central nervous system is often able to adapt itself to slowly increasing pressure, little or no hypothalamic dysfunction may be observed. Diabetes insipidus may sometimes occur and is attributable to pressure on the supraopticohypophysial tracts or the neurohypophysis. However, the importance of hypothalamic extensions is that acute and sometimes fatal disturbances of hypothalamic function may follow removal of a tumour.

Symptoms of involvement of the structures of the cavernous sinus may occur with benign pituitary tumours, and the oculomotor nerve is affected more often than the abducens and trochlear nerves. Very rarely the internal carotid artery is obstructed and ischæmia of the corresponding cerebral hemisphere results. Extension of chromophobe adenomas into the frontal or temporal regions may be silent, or produce localizing signs of lesions in these situations.

Chromophobe adenomas replace secreting cells and thus cause hypopituitarism. There is tiredness, mental inertia, sensitivity to cold, loss of libido, impotence in the male, amenorrhœa in the female, and loss of hair on the body, including

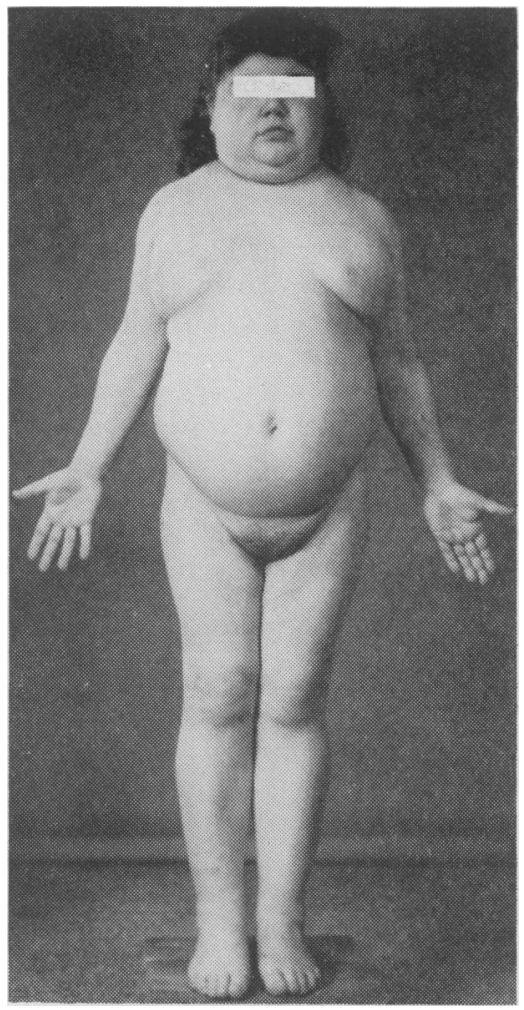

Fig. 3.-A girl of 12 years with Cushing's disease. Sireew has the typical 'moon' face and obesity. Other features were a flushed face, a blood pressure ofo $150 / 100 \mathrm{~mm}$. $\mathrm{Hg}$, and severe mental depression. There was no abnormality of the visual fields. Plain X-rays of the skull showed rarefaction of then posterior clinoid processes, and basal cisternography revealed a tumour similar to that seen in Fig. 4. Removal of the tumour produced a striking $\overrightarrow{\widehat{O}}$ improvement in her condition. Histological examination showed a mixed-celled pituitary tumour.

the axillæ and pubis. The hair of the head is fine and the skin feels thin and smooth, due to loss of $\mathrm{B}$. connective tissue. Patients suffering fromo hypopituitarism are usually not emaciated. Their. hypersensitivity to drugs (e.g. opiates andố barbiturates) and anæsthetics should be remembered when surgery is contemplated.

Acidophil adenomas or hyperplasia of the acidophil cells of the pituitary gland cause the endocrine picture of acromegaly. As previouslyñ stated, only a small minority of acidophil adenomas cause pressure on the optic nerves and chiasm. The cells of acidophil adenomas produce anc excess of the growth or somatotrophic hormone (STH) and the allied diabetogenic hormones Thus gigantism occurs if the epiphyses are stillo active and acromegaly if they are united, and ${ }^{\text {? }}$ diabetes mellitus is common. Sexual desire and potency may be reduced or abolished, presumably 


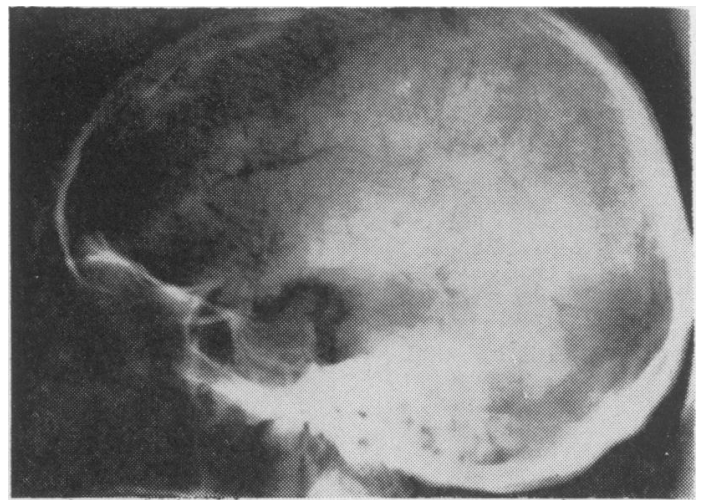

Fig. 4.-Basal cisternography revealing the upward and backward extension of a chromophobe adenoma.

by suppression of the basophil cells. When not caused by an expanding tumour, the abnormal endocrine secretion may abate or cease altogether. Removal is indicated when an adenoma causes pressure on the optic pathways. Some improvement in the endocrine state may follow operation but is not sufficient to warrant operation for this reason alone.

Basophil adenomas are rare. They are small and very rarely expand the pituitary fossa to compress the optic pathways. Only the occasional case of Cushing's disease (basophilism) is caused by a basophil adenoma (Fig. 3).

Craniopharyngiomas, or tumours of Rathke's pouch, cannot usually be regarded as favourable tumours except when they arise in the pituitary fossa and behave like chromophobe tumours.

\section{Radiography of Pituitary Tumours}

Almost all tumours which arise in the pituitary fossa below the sellar diaphragm cause ballooning of this structure. Its outline appears roughly circular in lateral X-ray views. The dorsum sellæ and posterior clinoid processes become thin and rarefied, and may finally disappear. The anterior clinoids are undermined or in various stages of destruction. Acidophil adenomas also produce signs in the skull associated with acromegaly (enlarged nasal air sinuses and mastoid processes, enlargement of the superciliary ridges, lower jaw and malar bones, and separation of the teeth). Occasionally, pituitary adenomas are entirely or almost entirely extrasellar. There is then no enlargement of the pituitary fossa. Craniopharyngiomas almost always show calcification of all or part of the cyst wall. When, as is usual, there is no evidence of raised intracranial pressure, air-encephalography should be carried out in patients with pituitary tumours to demonstrate the amount of extrasellar extension (Oliver, 1952). An attempt is made to fill the basal cisterns (cisternography) around the pituitary tumour and also the third ventricle (Fig. 4). On the rare occasions when the intracranial pressure is raised, ventriculography is carried out instead of encephalography. If signs of endocrine dysfunction are minimal or absent, bilateral carotid angiography is done to exclude an aneurysm of the anterior communicating artery or the internal carotid artery.

\section{The Temporal Region}

The temporal cortex is concerned with the reception, directly or indirectly, of associationfibre systems of the other areas of the cerebral cortex, and may therefore be expected to play an important part in the total function of the cerebral cortex. Nevertheless, personality changes are rarely associated with expanding lesions of the temporal fossa, but when they do occur they take the form of anxiety, irritability and occasionally aggressiveness. This is in marked contrast with the apathy often produced by expanding lesions of the anterior fossa. Memory and calculation may, however, be disturbed. Interference with the superior temporal convolution of the dominant hemisphere may cause nominal aphasia.

About one-quarter of all cases of epilepsy have their focus of discharge in one or other temporal lobe. Although atrophy is the most commonly occurring underlying pathological process, tumours account for a significant proportion of cases of temporal lobe epilepsy. Often the epileptic discharge is confined to the temporal lobe, giving rise to characteristic phenomena, but it may spread over the whole of the cerebral cortex to produce grand mal attacks.

There are several modes of origin of temporal lobe epilepsy and each reflects one of the functions of the temporal lobe. The commonest is a peculiar, indescribable sensation in the abdomen, chest or throat. This is the visceral aura and is related to autonomic function. The classical attack, originally described by Hughlings Jackson, begins with a sensation of a bad smell or taste accompanied by smacking of the lips and a 'dreamy state'; the origin of such attacks is in the uncus or hippocampus, the location of olfactory and gustatory function. Other patients complain of unpleasant noises or a disturbance of equilibrium, sometimes so severe that the patient is thrown to the ground; sounds may seem unduly loud or soft. These attacks are related to the auditory and equilibratory functions of the superior temporal convolution. Some patients experience sudden fear, anger or pleasure. There may be a sudden feeling of familiarity with the surroundings, or the patient may feel that what is happening has taken place before, the déjà vu phenomenon. Sometimes objects seem small and receding, or large and near. A vivid recollection of the past with its associated emotions may force its way into consciousness. Patients may carry out complicated acts unrelated to present events but sometimes related to the sensory phenomena and tending to be aggressive. This is not to be confused with post-epileptic automatism. Depersonalization, a feeling of complete detachment from self, and the related phenomenon of 
autoscopy (i.e. seeing an image of oneself in the external environment) are sometimes experienced. These curious effects are related to the highest functional levels of the temporal lobe.

Expansion of temporal lesions upward often causes contralateral facial paresis due to involvement of the facial area of the frontal cortex or its efferents. Expansion upwards and medially causes contralateral facial paresis and hemiparesis by interference with the homolateral pyramidal fibres.

The optic radiation sweeps widely forward in the upper posterior part of the temporal lobe before passing backward to the occipital cortex. Thus lesions within the temporal lobe may invade the optic radiation from below, and as light rays from above strike the lower part of the retina and light rays from one side strike the opposite side of the retina, contralateral homonymous upper quadrantic field defects are produced. This is in contrast with lesions within the parietal lobe, for they invade the optic radiation from above and cause contralateral homonymous lower quadrantic field defects. A lesion arising in either situation may eventually cut across the whole of the optic radiation and produce a complete contralateral homonymous hemianopia. Contralateral visual hallucinations are sometimes produced and may lead to the diagnosis of mental illness. Involvement of the optic radiation usually, though not invariably, indicates a destructive lesion within the cerebrum rather than compression of its surface by a pathologically benign lesion.

Several varieties of meningioma are found in the temporal fossa.

Medial sphenoid-ridge meningiomas produce a characteristic syndrome. Patients complain of unilateral loss of vision and sometimes unilateral exophthalmos. There is homolateral optic atrophy, diminished visual acuity, and, in the early stages, a central scotoma. There may be papillodema on the other side (Foster-Kennedy syndrome). When the optic tract is involved instead of the optic nerve, there is an homonymous hemianopia. Ophthalmoplegia is present in the majority of cases, and results from occlusion of the orbital fissure. The ophthalmic division of the trigeminal nerve is affected in some patients causing paræsthesia and diminished sensation over the forehead. Temporal lobe epilepsy may occur, and is sometimes the presenting symptom. These deeply placed tumours tend to envelop the internal carotid artery and to adhere to the corresponding optic nerve.

Middle sphenoid-ridge meningiomas usually grow to large dimensions before revealing themselves. Manifestations of raised intracranial pressure may be associated with personality changes produced

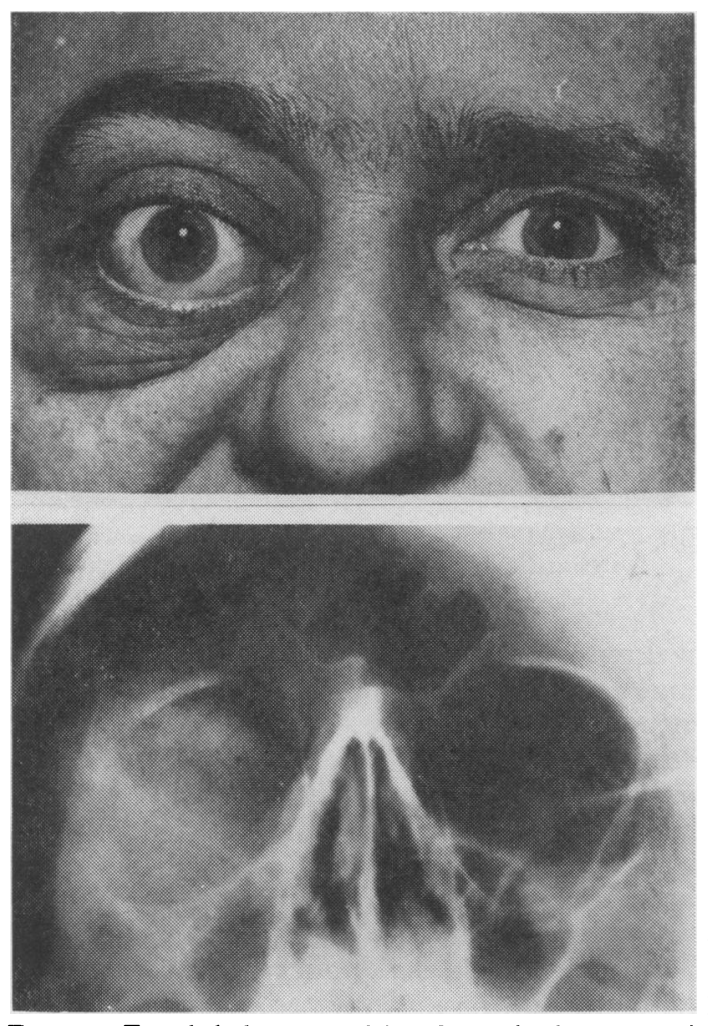

Fig. 5.-Exophthalmos resulting from the hyperostosis produced by a meningioma of the sphenoid ridge.

by upward extension into the frontal region. Sometimes the presenting symptom is unilateral exophthalmos. Plain radiography may show hyperostosis or erosion of the lesser wing of the sphenoid. Occasionally the hyperostosis is widespread, involving much of the orbit and middle fossa (Fig. 5).

Lateral sphenoid-ridge meningiomas produce few or no localizing signs. Such tumours on the dominant side may cause memory defects or nominal dysphasia. Radiography may reveal hyperostosis or erosion of bone.

Meningiomas en plaque are tumours which lie like a carpet over the dura mater, and are characterized by an increase in thickness of the adjacent bone and lack of neurological effects. The tumour most often grows in relation to the sphenoid bone and runs a chronic course. The presenting symptom is unilateral exophthalmos which again is produced by thickening of the bony walls of the orbit.

Sylvian fissure meningiomas are attached to the dura mater overlying the posterior ramus of the Sylvian fissure, and penetrate between the frontal and temporal lobes. Localizing evidence may be found; it includes personality changes, contra- 

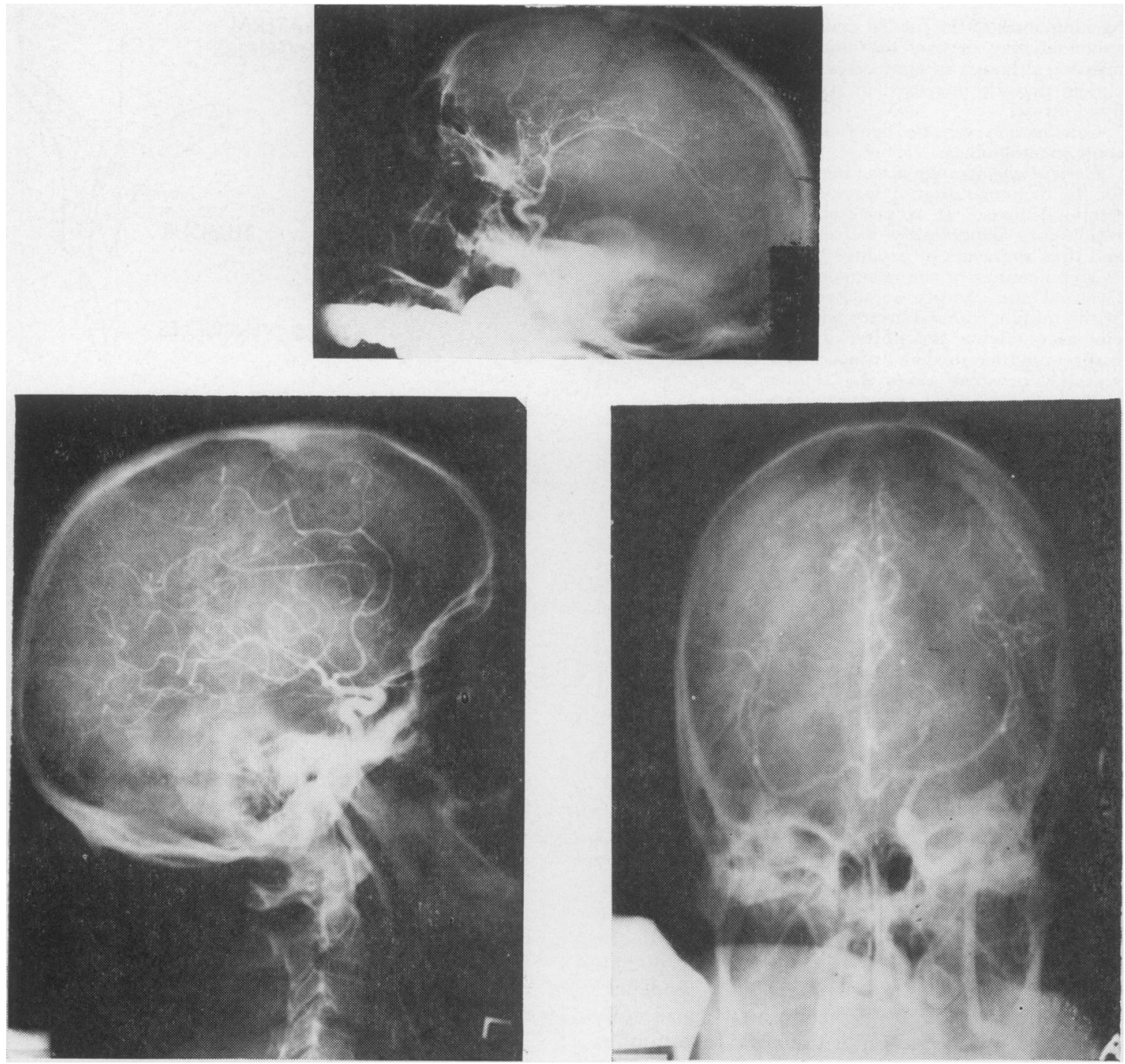

Fig. 6.-Above, the upward curve of the middle cerebral artery and its branches produced by a tumour in the temporal region, in this case a meningioma of the sphenoid ridge.

Below, on the left, a lateral carotid angiogram that might be considered normal. However, the clinical evidence was strongly in favour of a temporal tumour, therefore an injection of contrast was given while the opposite carotid artery was compressed. The simultaneous filling of both middle cerebral arteries in the Towne's projection shows elevation of the one on the left proving the presence of a temporal tumour. (Angiography by Dr. G. F. Swann.)

lateral facial paresis, Jacksonian attacks beginning in the face, and nominal dysphasia with tumours on the dominant side.

\section{Investigations}

What I have previously said about lumbar puncture applies with particular force with tumours located in the temporal region.

Plain radiography may show bulging of the temporal fossa and/or elevation of the lesser wing of the sphenoid with a long-standing lesion which has developed during childhood. If calcified, the pineal gland may be seen to be displaced away from the side of a tumour. Increased width and tortuosity of vascular channels, occasionally with enlargement of the foramen spinosum, may be seen with meningiomas. Sometimes there are adventitious vascular channels converging towards the site of a meningioma.

Increase in thickness of the lesser and greater wings of the sphenoid, the roof and lateral wall of the orbit, occurs with most meningiomas of the lesser wing of the sphenoid and meningiomas en plaque. As with tumours elsewhere within the cranium, rarefaction or total destruction of the posterior clinoid processes may be caused by long-standing raised intracranial pressure. 
Meningiomas of the medial end of the lesser wing of the sphenoid may destroy the clinoid processes by direct invasion although in some cases the homolateral anterior clinoid may be increased in size, a special example of hyperostosis.

Calcification can be demonstrated radiologically in some meningiomas.

Carotid angiography is the most valuable investigation for the demonstration of space-occupying lesions in the temporal fossa. It is preferable to ventriculography which may dangerously increase intracranial pressure and thus aggravate or produce brain-stem compression.

Meningiomas of the sphenoid ridge tend to produce localized and sharply angulated upward displacement of the middle cerebral artery and its branches. Meningiomas overlying the posterior ramus of the Sylvian fissure produce medial displacement or folding of the vessels. In some cases the tumour gives rise to a visible circumscribed vascular pattern.

Space-occupying lesions within the temporal fossa produce a characteristic upward curve of the whole of the middle cerebral artery and its branches, best seen in lateral projections (Fig. 6).

Ventriculography is avoided whenever possible in the diagnosis of expanding lesions in the temporal fossa for the reasons already given, but may be required when lateralizing signs are not obtained or if angiography is unsatisfactory. A-P views show displacement of the ventricles towards the opposite side with angulation of the third ventricle on the septum lucidum (Fig. 7). Lateral views taken after manœuvres to fill the temporal horns may demonstrate absence of filling, deformity or displacement of the temporal horn. Lateralizing evidence may sometimes be obtained by means of electroencephalography.

\section{The Parieto-occipital Region}

The Parieto-occipital Syndrome. Bizarre subjective sensory phenomena, referred to the opposite side of the body, may be produced by lesions of the sensory area of the cerebral cortex. Patients sometimes complain of formication (sensation of ants creeping on the skin) on one side of the body. These strange sensory phenomena are periodic and are probably epileptic. Indeed they may form the aura of more fully developed fits. Invasion of the optic radiation (as also with tumours of the temporal lobe) may produce visual hallucinations, whereas lesions which affect the visual cortex cause unorganized visual sensations (flashes of light). It is easy to understand why patients with parietal lobe tumours may be referred to a psychiatrist by mistake. When tumours arise in the dominant parietal lobe, speech is affected. Destruction of the angular gyrus produces the syndrome of alexia (inability to read) and an homonymous hemianopia. A destructive lesion further forward, affecting the supra-marginal gyrus, causes global (i.e. total) aphasia. A tumour on the non-dominant side may cause a defect of body image so that the patient becomes unaware of the opposite side of the body (corporeal agnosia).

There may be disturbances of sensation (diminished or absent sensation of light touch,

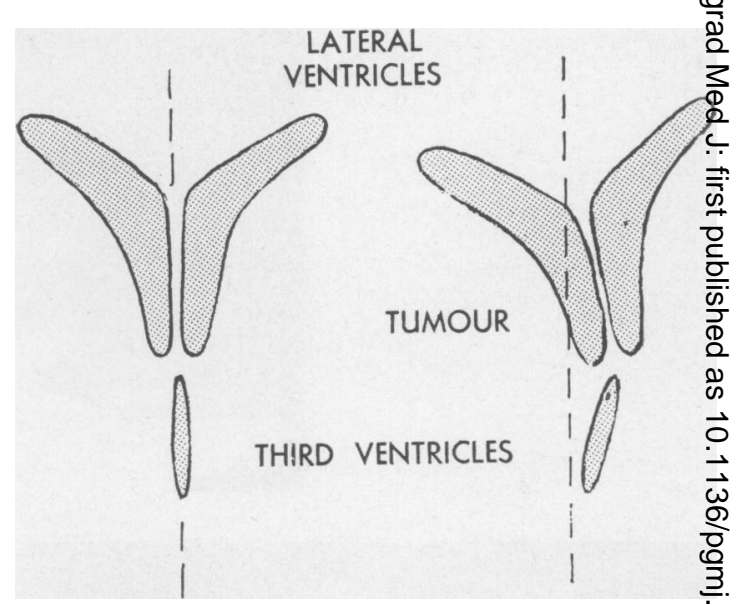

FIG. 7.-Drawing of a ventriculogram showing on the right the angulation of the third ventricle and septum lucidum commonly produced by temporas tumours. (From 'Basic Surgery', edited by Leslig, Oliver. London: H. K. Lewis.)

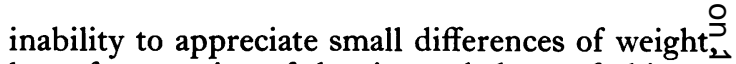
loss of perception of the size and shape of objectscs i.e. astereognosis or tactile agnosia). The receptiones zone for stereognosis is widespread in the parieta $\Phi$ lobes. Visual agnosia (inability to recogni objects when vision is intact) is sometimes fougad with posterior parietal lesions on the domininte side. Apraxia (inability to perform purposefue acts when the pyramidal pathways are intact) sometimes observed with parietal lesions.

A tumour extending downwards or backwards interrupts the optic radiation, affecting the upper most fibres first. Therefore, homonymous lowero quadrantic field defects are produced, in contras? with temporal tumours which affect the optie radiation from below and cause homonymous upper quadrantic defects. When both the upper and lower parts of the optic radiation are affected by tumours, there is hemianopia with sparing of macular vision, although complete division of the optic radiation, as in removal of the occipital lobe? causes complete homonymous hemianopia, in cluding transection of the macular part of the field Visual attention is sometimes lost with lesions of the parieto-occipital lobe, and attention to other forms of sensory stimuli may also be defective? Thus light touch may be felt equally well on both sides of the body when they are testeds separately, whereas simultaneous stimulation of both sides together results in extinction of appreciation of the stimulus on the side opposite to the lesion. In a normal person, the sudde approach of an object towards the eyes cause blinking. This blink reflex may be absent wher? objects approach the eyes from the side opposite to a parietal lesion. A tumour extending anteriorlyo 


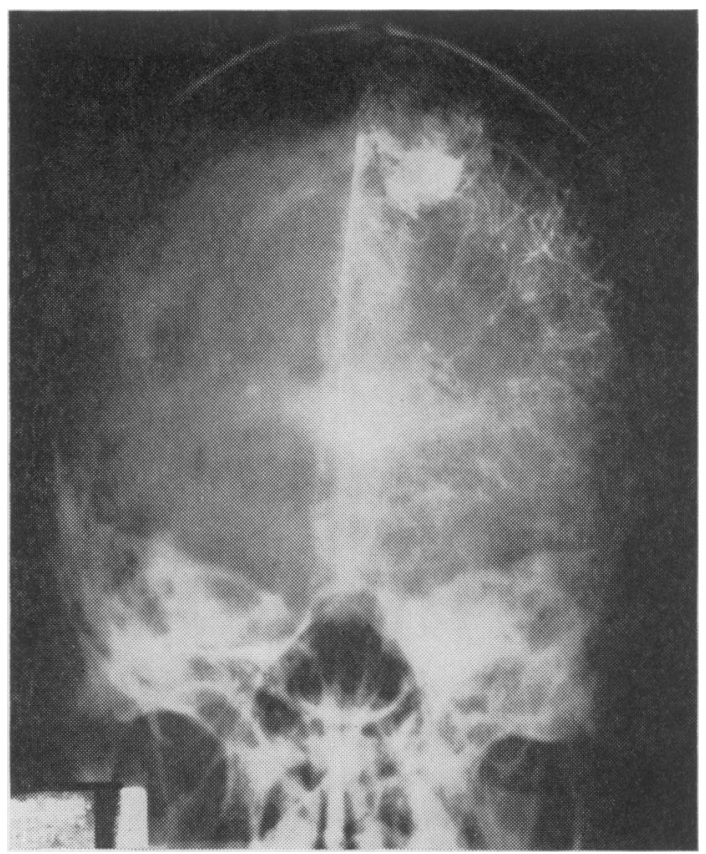

FIG. 8.-Angiogram showing the vascular pattern of a parasagittal meningioma. A man, aged 45, had had recurrent headaches on the same side for many years and attacks of tingling and clonic movements of the opposite foot for six months; the foot was also weak. The neurological state was normal four months after removal of the tumour. (Angiography by Dr. G. F. Swann.)

involves pyramidal fibres causing contralateral paresis. Because of the inclination of the motor cortex the arm and leg are more likely to be affected than the face.

\section{Investigations}

Angiography. Parietal tumours tend to cause downward displacement of the terminal branches of the middle cerebral artery. There may also be a vascular pattern (Fig. 8). The angiogram, however, may be entirely negative, as some of these tumours lie towards the termination of the main cerebral arteries.

Ventriculography. Anteriorly-placed tumours show deformity of the body of the lateral ventricle, whereas posteriorly-placed tumours show deformity and displacement of the occipital horn. There may also be a general displacement of the ventricles to the opposite side.

\section{Pathology of Parieto-occipital Tumours}

Meningiomas. Parasagittal meningiomas are common in this region, especially anteriorly where they involve the motor as well as the sensory cortex (Hoessly and Olivecrona, 1955). Because the motor area for the lower extremity is on the medial surface of the hemisphere, the leg tends to be involved before the arm and face. Bilateral parasagittal meningiomas cause weakness of both

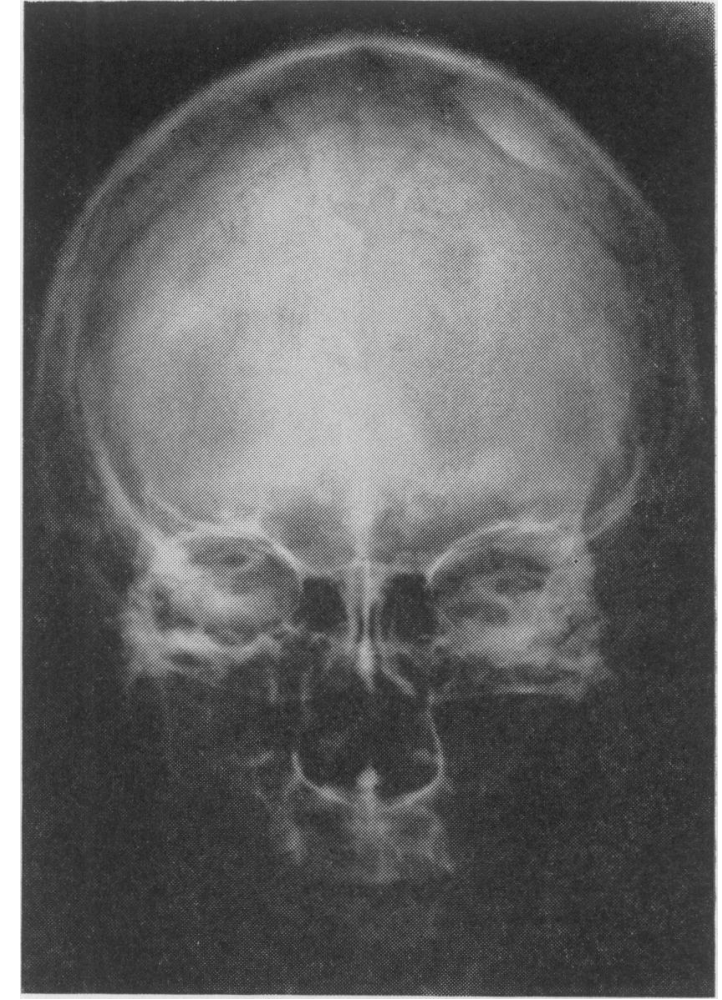

FIG. 9.-An area of hyperostosis overlying a parietal meningioma.

lower extremities. Plain radiography may show: increased vascular markings; hyperostosis (Fig. 9); erosion of bone; calcification of the tumour, but often there is no evidence in the plain films to indicate a meningioma.

At operation, tumours arising from the falx are likely to be concealed by the brain, whereas those arising from the superior longitudinal sinus are encountered immediately after reflection of the dura mater. Some meningiomas in this region arise from the dura over the surface of the parietal lobe without involving the superior longitudinal sinus. Ablation of all but the very smallest meningiomas in this situation should begin with piecemeal removal of the interior of the tumours. The capsule then collapses away from the brain and damage to the motor and sensory cortex is thus avoided. Great care must be taken to prevent injury to veins draining into the superior longitudinal sinus. If a tumour is attached to the sinus, a thin layer of tumour tissue is left behind, for the sinus must not be damaged in this situation. Careful electrocoagulation of the remaining tissue is advisable.

Peritorcular meningiomas are rare. They may arise from any of the four quadrants around the 


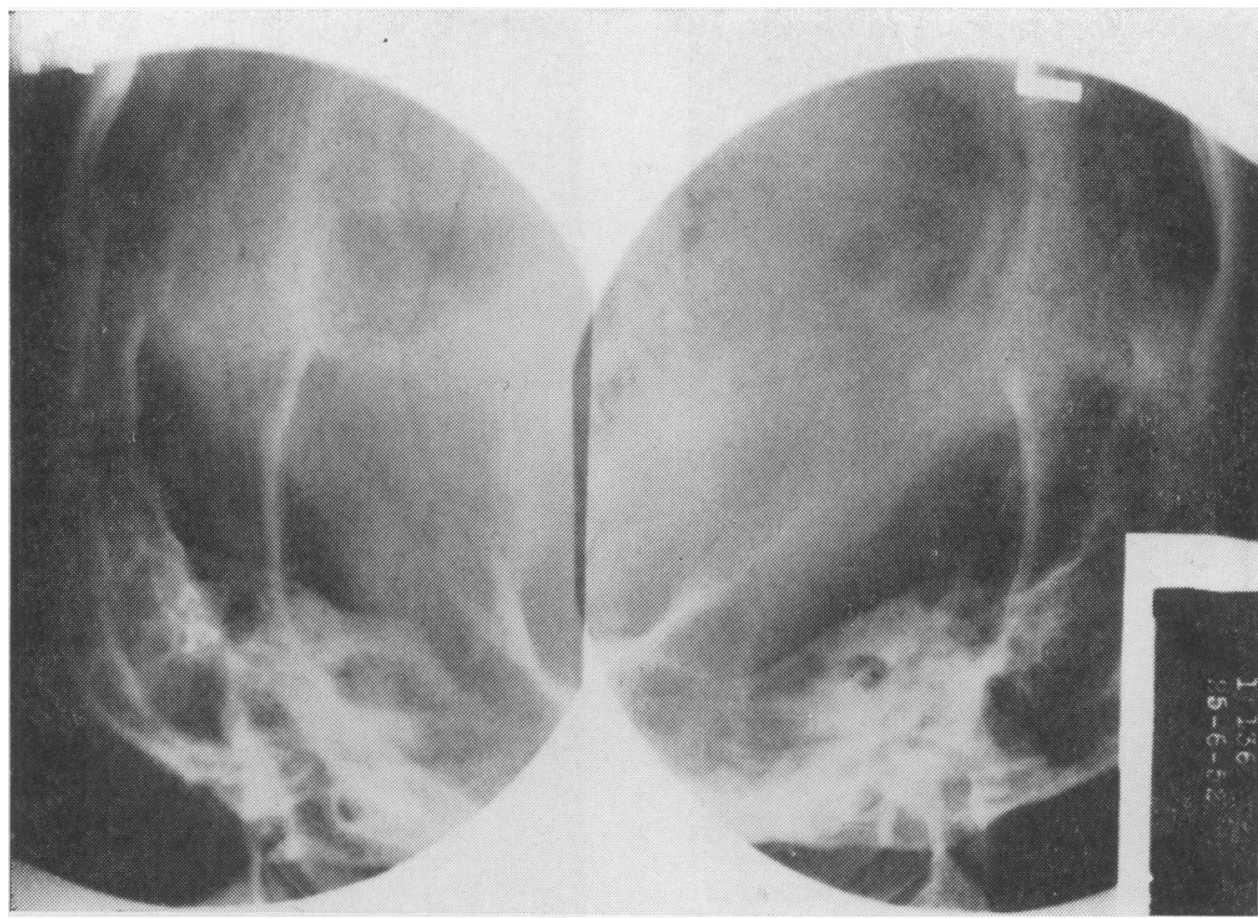

Fig. I ).-On the reader's right, a normal internal auditory meatus.

On the reader's left, the meatus and canal have been enlarged by the growth of an acoustic tumour.

venous confluence. Some cause homonymous visual field defects whereas others produce cerebellar signs. These tumours have a tendency to recur. The approach is by means of an occipital flap based on the occipital muscles.

\section{The Posterior Cranial Fossa}

One or more of several effects may be brought about by expanding lesions of the posterior fossa. The flow of cerebrospinal fluid through the aqueduct and fourth ventricle may be obstructed and the resulting hydrocephalus is revealed by frontal headache, vomiting and papillodema. Sub-occipital headache occurs in some cases in association with a cerebellar pressure cone in the foramen magnum.

Unilateral or bilateral external rectus paralysis occurs with some tumours in the posterior fossa due to stretching of the abducens nerves. This is brought about by descent of the brain-stem produced by increasing pressure in the ventricles above the tentorium.

A tumour expanding from a lateral position towards the midline may cause lateral nystagmus, which is slower and of greater amplitude when the eyes look towards the side of the lesion: hypotonia, incoordination, intention tremor, dysdiadochokinesia and past-pointing may demonstrated in the arm on the side of the tumoup and the outstretched arm may deviate spon? taneously outwards and downwards. Sometimes there is hypotonia and incoordination of tho homolateral leg and veering or falling towards the side of the tumour. A tumour expanding in the midline behind the brain-stem tends to causê trunk ataxia and hypotonia of the lower limbs.

Pressure may occur on pyramidal fibres of either or both sides. Contralateral pyramida signs are produced by direct pressure of the tumour: on the brain-stem, whereas homolateral pyramida $\$$. signs are produced by the cerebral peduncle of the opposite side being forced against the free edge of the tentorium cerebelli and consequent pressure on pyramidal fibres which cross below in the medulla oblongata to the side of the lesiono Extrinsic tumours are likely to compress the cranial nerves of the posterior fossa early in theip evolution, whereas intrinsic tumours tend to compress them late, or not at all. For reasons a\& yet unexplained, cranial nerve palsies occasionally occur on the side opposite to the tumour. Severe compression of the medulla oblongata may occue from an expanding tumour arising anywhere in the posterior fossa, and may give rise to vertigo dysarthria, dysphagia, repeated vomiting, brady $\overrightarrow{0}$ 

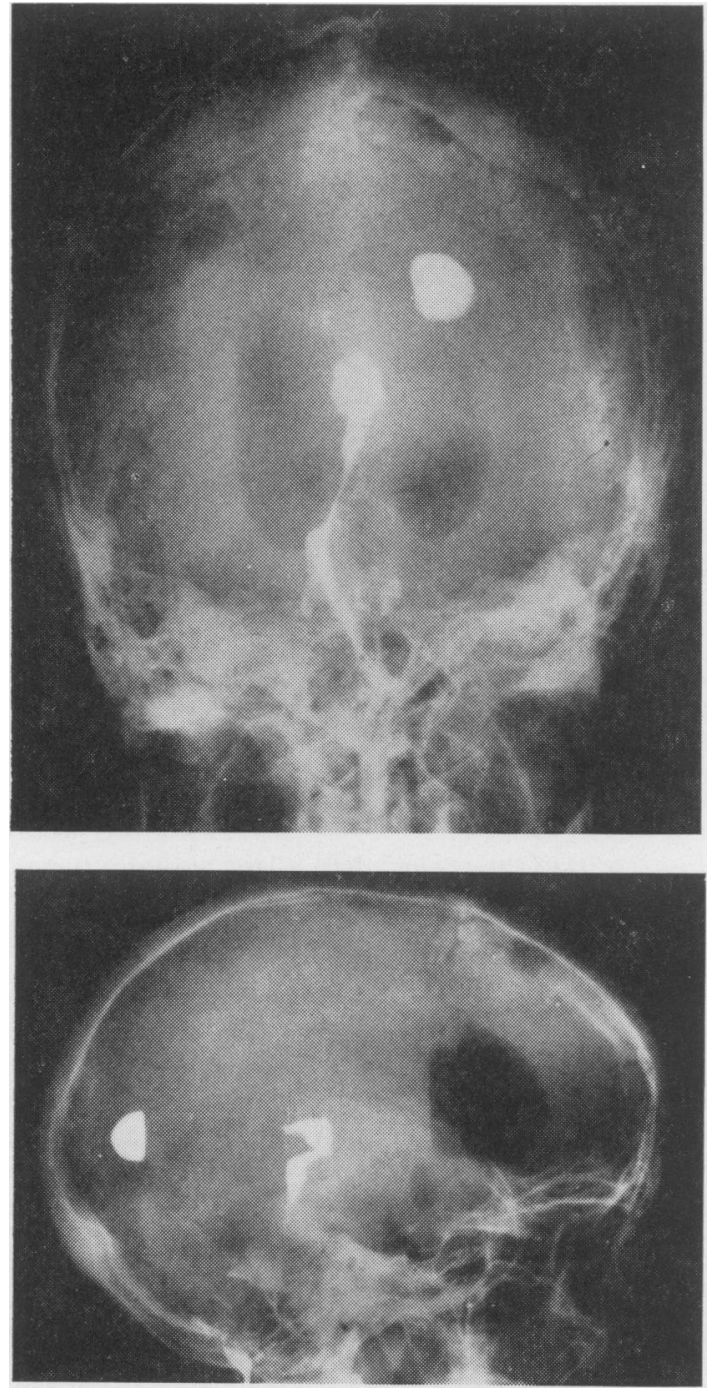

FIG. I1.-Positive and negative contrast ventriculography in a patient with an atypical acoustic tumour syndrome. Air shows dilatation of the lateral ventricles.

Above, Myodil shows dilatation of the third ventricle and upper part of the aqueduct, constriction of the lower part, and characteristic rotation of the fourth ventricle.

Below, Myodil shows forward bending of the aqueduct and forward displacement of the fourth ventricle.

cardia, slowing of respiration and eventually Cheyne-Stokes respiration.

Bilateral inferior temporal scotomas or bitemporal lower quadrantic field defects (never a complete bitemporal hemianopia) may result from pressure on the optic chiasm by the anterior end of the distended third ventricle.

Slowly expanding intrinsic cerebellar tumours (e.g. astrocytomas and hæmangioblastomas) quite often cause little or no cerebellar disturbance and give no evidence of involvement of cranial nerves or the brain-stem, but present with manifestations of raised intracranial pressure alone. Such tumours are easily mistaken for 'silent' tumours arising in other parts of the cranium (e.g. prefrontal and pretemporal regions). Furthermore, frontal and even parietal lesions occasionally produce mild contralateral 'cerebellar' signs. Rapidly expanding lesions, such as cystic tumours and cerebellar hæmorrhage, are liable to cause acute medullary compression and acute hydrocephalus.

\section{Investigations}

Plain X-rays may show evidence of long-standing raised intracranial pressure, as previously discussed in relation to tumours elsewhere in the cranium, or they may show localizing evidence of a tumour, as with most acoustic tumours (Fig. 10).

Ventriculography usually reveals dilatation of the lateral ventricles and third ventricle. Displacement, deformity and dilatation of the aqueduct and fourth ventricle may also be demonstrated. Tumours in the posterior fossa sometimes cause upward displacement of the third ventricle, or upward curving of the posterior horn of the lateral ventricle on the side of a laterallyplaced tumour. Marked indentation of the occipital horn occurs with dumb-bell meningiomas arising from the tentorium and growing supra- and infra-tentorially. Positive contrast ventriculography with Myodil ( 1 to 2 ml.) injected through the anterior horn of one of the lateral ventricles gives more reliable information regarding the aqueduct and fourth ventricle than air ventriculography (Fig. I I). However strong the clinical evidence of a posterior fossa tumour may be, ventriculography should always be done except, perhaps, when there is indisputable evidence of an acoustic tumour, which usually produces a characteristic syndrome. Vertebral angiography is more dangerous than carotid angiography. Therefore it is not employed if the necessary information can be obtained otherwise. Aneurysms, vascular malformations and vascular tumours are outlined in the angiograms.

\section{Types of Benign Tumours}

Astrocytomas are the commonest intrinsic cerebellar tumours. The maximum incidence is during the first two decades. In the cerebellum most astrocytomas are Grade I, i.e. the most benign type, and most of them are cystic (Mabon, Svien, Adson and Kernohan, 1950). Frequently there is a large cyst with a small mural tumour. The cystic fluid is yellow and contains a large amount of protein. Removal of the mural nodule is usually all that is required.

Hamangioblastomas (Syn. Angioreticulomas). These tumours occur most often in the cerebellum; they are rare elsewhere in the nervous system. They are more often cystic than solid. The cysts tend to be large and contain mural tumours like those in cystic astrocytomas of the cerebellum. Unlike the latter tumours, however, hæman- 
gioblastomas are found most often in adults, and they show themselves earlier in women than in men. Thus in women the maximum incidence occurs between 20 and 40 years, whereas in men it is between 40 and 60 years. Sometimes these tumours are familial; they then tend to be multiple. Therefore vertebral angiography may be advisable for suspected hæmangioblastomas when there is a family history of these tumours, otherwise multiple tumours may be overlooked at operation (Olivecrona, 1952). The association with hæmangioblastomas elsewhere and cysts in the liver, pancreas or kidney, is known as Lindau's syndrome. Angioma of the retina (von Hippel's disease) may be one of the lesions forming part of Lindau's syndrome.

Acoustic Nerve Tumours (Syn. Acoustic Neurinomas). These tumours are thought to arise from the endoneurium of the acoustic nerve within the internal auditory meatus. They grow in the subdural space, and as they expand come into relationship with the trigeminal nerve, cerebellum, pons, and eventually also with the ninth, tenth, eleventh and twelfth cranial nerves. They are usually solid tumours but are commonly surrounded by a cyst formed by the arachnoid. Bilateral acoustic tumours are sometimes found in the 'central' type of von Recklinghausen's neurofibromatosis. Acoustic tumours are composed of interlacing bundles of spindle cells containing elongated nuclei. The cells tend to be arranged in rows ('palisading') and some form whorls around blood vessels. Reticulum fibrils are conspicuous between cells but nerve fibres are rarely seen (Russell, 1950).

The description of these tumours and their symptoms was first adequately given by Harvey Cushing (1917).

They affect both sexes equally, and symptoms begin in middle age and have usually been present for two to three years before the neurosurgeon is consulted. The first symptom is gradually progressive deafness in one ear, frequently accompanied by tinnitus and dizziness; severe vertigo is rare. Involvement of the vestibular division of the eighth cranial nerve is demonstrated by the caloric test.

Paræsthesia in the distribution of the fifth cranial nerve is common, but trigeminal pain is rare. Absence of the corneal reflex may be the only sign of involvement of this nerve. Strangely enough, there is usually little or no evidence of compression of the facial nerve. There may be aching pain in the occipital muscles and tenderness behind the homolateral mastoid process. Later there are cerebellar signs. In some cases, compression of the brain-stem produces pyramidal signs on either or both sides. Later there are

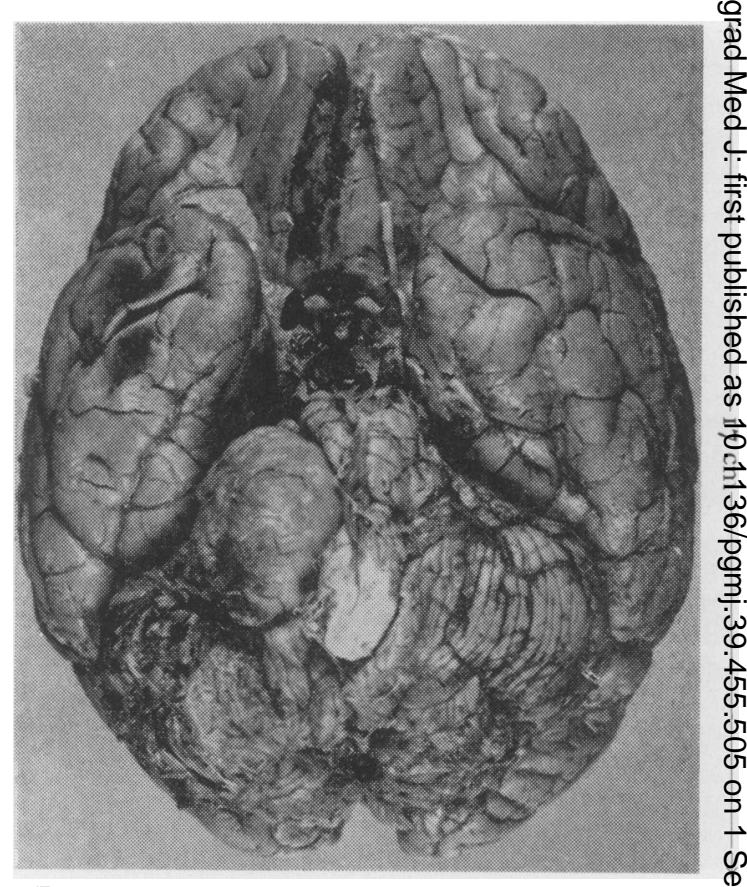

Fig. 12.-On the reader's left, a large acoustic tumo $\frac{\mathbb{D}}{\mathbb{R}}$ is shown severely indenting the brain-stem. (From 'Basic Surgery', edited by Leslie Oliver. Londore H. K. Lewis.)

signs of increased intracranial pressure (headaç vomiting and papillœdema, abducens nerve pæa lysis). Extension of the tumour to the tenth an eleventh cranial nerves causes unilateral palata栾 pharyngeal and laryngeal paralysis and als partial paralysis of the sternomastoid and trapeziug muscles.

Radiography often shows enlargement of the interna auditory meatus or sometimes considerable erosion $\vec{f}$ the petrous bone (Fig. 10). When the syndrome atypical, positive contrast ventriculography (Myodif I to $2 \mathrm{ml}$.) is carried out to show the aqueduct and fourth ventricle (Fig. I I).

Removal of acoustic tumours is carried out throug a posterior fossa approach with the patient in the sitting position. Operation still has a considerable mortalif (up to $25 \%$ ) because these tumours are usually referrea to the neurosurgeon when there is marked indentation of the brain-stem and advanced hydrocephalus (Fig. 1 $2 f$ Decompression alone is valueless, for these tumours art firmly attached to the petrous bone and therefore the pressure on the brain-stem is not relieved. The facial nerve is often not seen during operation, and facifis palsy commonly results. It is treated by facial-hypöglossal or facial-accessory anastomosis. Also intrao cranial grafting can be done if enough of the faciaf nerve is left at the pontine end (Dott, 1958).

Meningiomas in the posterior fossa may be attached to any part of the dura mater, buf usually arise somewhere along the transverse or sigmoid sinuses (Cushing, 1938). Sometimes the 
grow in the cerebello-pontine angle and mimic acoustic tumours. Dumb-bell meningiomas of the tentorium are rare tumours which grow upward to indent the parieto-occipital lobe and downward into the posterior fossa. In the removal of meningiomas of the posterior fossa, the transverse sinus on one side may be divided or excised when necessary, as there is usually an anastomotic channel between the two transverse sinuses. The presence of this connection may, however, be verified pre-operatively by venography (the superior longitudinal sinus is injected through a burr-hole with $45 \%$ hypaque solution and rapid serial X-rays are taken.

Epidermoid Tumours (Syn. Cholesteatomas, Pearly Tumours of Cruveilhier). These are rare congenital tumours arising from the meninges and consisting of a capsule of epidermal tissue enclosing a mass of epithelial debris and cholesterol crystals. They are not to be confused with the inflammatory type of 'cholesteatoma' caused by chronic infection of the middle ear and mastoid. The whole of the contents of the 'cyst' and as much of the capsule as possible must be removed, for if some cholesterol remains it causes an aseptic meningitis which can be fatal.

In conclusion, I wish to emphasize again that any patient suspected of harbouring an intracranial tumour should be thoroughly examined and investigated with all possible speed and the histological nature of the lesion determined. Neurosurgical facilities, including ventriculography and angiography, should be available at any time of the day and night, for sometimes tumours produce acute symptoms that may need immediate relief. There will be many disappointments but failure to apply a rigid discipline and to maintain an attitude of urgency in the management of tumour suspects will lead to the missing of favourable lesions.

\section{REFERENCES}

Balley, P. (1948): 'Intracranial Tumours'. Springfield, Illinois: Charles C Thomas.

Cushing, H. (1917): 'Tumours of the Nervus Acusticus and the Cerebello-pontine Angle'. Philadelphia and London: Saunders.

- and EisenhardT, L. (1938): 'Meningiomas'. Springfield, Illinois: Charles C Thomas.

Davis, L., Martin, J., Padberg, F., and Anderson, R. K. (1950): A Study of 182 Patients with Verified Astrocytoma, Astroblastoma and Oligodendroglioma of the Brain, $\mathcal{F}$. Neurosurg., 7, 299.

Dotт, N. M. (1958): Facial Paralysis: Restitution by Extra-petrous Nerve Graft, Proc. roy. Soc. Med., 51, 9oo.

Earnest, F., Kernohan, J. W., and Craig, W. McK. (1950): Oligodendrogliomas, Arch. Neurol. Psychiat. (Chicago), 63,964 .

Henderson, W. R. (1939): The Pituitary Adenomata (Dr. Harvey Cushing's Series), Brit. F. Surg., 26, 8r r.

Hoessly, G. F., and Olivecrona, H. (1955): Report on 280 Cases of Verified Parasagittal Meningioma, $\mathcal{F}$. Neurosurg., I2, 6r4.

JefFerson, G. (1950): Tumours of the Frontal Lobe, Postgrad. med. F., 26, 133.

, and JACKson, H. (1939): Tumours of the Lateral and of the Third Ventricle, Proc. roy. Soc. Med., 32, I ro5.

Mabon, R. F., Svien, H. J., Adson, A. W., and Kernohan, J. W. (1950): Astrocytomas of the Cerebellum, Arch. Neurol. Psychiat. (Chicago), 64, 74.

Olivecrona, H. (1952): The Cerebellar Angioreticulomas, $\mathcal{F}$. Neurosurg., 9, 317.

Oliver, L. C. (1952): 'Essentials of Neurosurgery'. London: H. K. Lewis.

Pennybacker, J. (1961): The Treatment of Pituitary Tumours, Proc. roy. Soc. Med., $54,619$.

Russell, D. S. (1950): The Pathology of Intracranial Tumours, Postgrad. med. $\mathcal{F}$., 26, rog.

SchmidT, M. B. (1902): Ueber die Tacchioni'schen Granulationen und ihr Verhältnis zu den Sarconen und Tsammomn der Dura Mater, Virchows Arch. path. Anat., 170, 429. 